\title{
Estimating One's Own and One's Relatives' Multiple Intelligence: A Study from Argentina
}

\author{
Adrian Furnham ${ }^{1}$ and Tomas Chamorro-Premuzic ${ }^{2}$ \\ ${ }^{1}$ University College London \\ ${ }^{2}$ Goldsmiths College London
}

Participants from Argentina $(N=217)$ estimated their own, their partner's, their parents' and their grandparents' overall and multiple intelligences. The Argentinean data showed that men gave higher overall estimates than women $(M=110.4$ vs. 105.1$)$ as well as higher estimates on mathematical and spatial intelligence. Participants thought themselves slightly less bright than their fathers ( 2 IQ points) but brighter than their mothers (6 points), their grandfathers (8 points), but especially their grandmothers (11 points). Regressions showed that participants thought verbal and mathematical IQ to be the best predictors of overall IQ. Results were broadly in agreement with other studies in the area. A comparison was also made with British data using the same questionnaire. British participants tended to give significantly higher self-estimates than for relatives, though the pattern was generally similar. Results are discussed in terms of the studies in the field. Keywords: multiple intelligence, self-estimation, other-estimation

Participantes de Argentina $(N=217)$ estimaron su propia inteligencia global y múltiple, así como las de su pareja, sus padres y sus abuelos. Los datos argentinos mostraron que los varones proporcionaron estimaciones globales más altas que las mujeres $(M=$ 110.4 vs. 105.1) además de estimaciones más altas en inteligencia numérica y espacial. Los participantes se percibían algo menos inteligentes que sus padres (2 puntos del $\mathrm{Cl}$ ) pero más inteligentes que sus madres ( 6 puntos), sus abuelos ( 8 puntos), y, en especial, sus abuelas (11 puntos). Las regresiones mostraron que los participantes creían que el $\mathrm{Cl}$ verbal y numérico eran los mejores predictores del $\mathrm{Cl}$ global. En general, los resultados eran similares a otros estudios del área. También se compararon los datos británicos con el mismo cuestionario. Los participantes británicos tendían a adjudicarse estimaciones significativamente más altas que a sus parientes, aunque, en general, el patrón era similar. Se comentan los resultados en términos de estudios en el mismo área.

Palabras clave: inteligencia multiple, auto-estimación, estimación de familiares

Reprint requests should be directed to: Professor A. Furnham, Department of Psychology, University College London, 26 Bedford Way, London WC1H OAP (United Kingdom). E-mail: a.furnham@ucl.ac.uk 
This is one of a series of programmatic studies examining primarily sex differences in self-estimates of multiple intelligence. Over the last decade, there have been a number of studies on the self-estimation of intelligence (Beloff, 1992; Bennett, 1997; Betsworth, 1999; Furnham, 2001; Furnham, Hosoe, \& Tang, 2001; Furnham, Shahidi, \& Baluch, 2000; Hogan, 1978; Paulus, Lysy, \& Yik, 1998; Reilly \& Mulhern, 1995) in many different countries (Yuen \& Furnham, 2004). Most studies have examined sex differences and shown consistent effects with women tending to give lower scores than men (Byrd \& Stacey, 1993; Furnham \& Gasson, 1998; Furnham, Clark, \& Bailey, 1999; Furnham, Fong, \& Martin, 1999, Rammstedt \& Rammsayer, 2002).

In the first study in the area, Hogan (1978) reported 11 studies, in half of which the difference between male and female self-estimations of IQ was statistically significant. Hogan contended that women's' tendency to perceive themselves as less intelligent than men is due to their denial of intellectual equality, which is socially rewarded and promotes better relations with men. Furthermore, he found that the majority of participants attributed higher IQ scores to their fathers than their mothers. Beloff (1992) offered a similar interpretation for her findings that women underestimate their intelligence whereas men overestimate it in a sample of 767 Scottish students. She proposed that in women's upbringing, there is an emphasis on humility and they receive "modesty training," resulting in poor intellectual self-image relative to men. Although no difference in self-estimates was found by Byrd and Stacey (1993) in New Zealand, estimates of family members' IQs revealed that women judged themselves to be equivalent to their sisters in terms of intelligence, whereas men perceived themselves as superior. However, a replication with a much larger sample showed there was indeed a male hubris-female humility effect in New Zealand (Furnham \& Ward, 2001).

Further studies looking at estimates of parental, grandparental, and sibling IQ appear to indicate that lay people believe that their fathers are more intelligent than their mothers (Byrd \& Stacey, 1993), their grandfathers more intelligent than their grandmothers (Furnham \& Rawles, 1995), and their brothers more intelligent than their sisters (Furnham, Fong, et al., 1999). People seem to believe every generation to be $1 / 3$ of a standard deviation more intelligent than the preceding one. Certainly, this may be due to the fact that men formerly attained higher educational qualifications than women. However, this is unlikely as an explanation for the very robust difference effect which has been shown to hold for school children and students with identical educational level (Furnham, 2001).

One reason for the belief that IQ rises with each generation may be that people are becoming aware of the Flynn effect. Flynn $(1987,1999)$ demonstrated "massive IQ gains" in 14 nations over time and it is possible that the popularization of this phenomenon in the media (as well as in institutions of higher learning) means that this influences people's estimates.

The expanding literature in this area covers a number of quite specific topics. Nearly 20 studies have examined sex differences in self-ratings of overall $I Q$, nearly all of which have shown a sex difference of 4 to 9 points. Men rate their own IQ higher than do women. Other studies have looked at sex differences in the ratings of relatives, specifically grandparents, parents, siblings, and children (Furnham, 2001). They show a consistent sex difference with female relatives being rated as less intelligent than male relatives. Furthermore, people seem to believe there are distinct generational differences, with each generation becoming more intelligent than the past generation (the Flynn effect).

Inevitably, there have also been a number of studies that have examined the relationship between self-estimated and psychometrically measured IQ (Borkenau \& Liebler, 1993; Furnham \& Fong, 2000; Furnham \& Rawles, 1999; Paulus et al., 1998; Reilly \& Mulhern, 1995;). The results show that when outliers are removed the correlations are typically in the range of $r=.25$ to $r=.50$ (Furnham \& ChamorroPremuzic, 2004).

As well as rating overall intelligence, a number of studies have examined estimates of specific types of intelligence such as emotional intelligence (Petrides \& Furnham, 2000), "successful" intelligence, and multiple intelligence as defined in 1983 and 1999 by Gardner (Furnham, Fong, et al., 1999; Furnham, Rakow, Sarmany-Schiller, \& De Fruyt, 1999). Most studies on self-estimates of intelligence involve measuring overall intelligence or "g." However, many researchers have made distinctions between various types of intelligence, an approach consistent with the view of most lay people. Gardner (1983) initially identified seven subtypes of intelligence that every normal individual should develop to some extent, but owing to a combination of heredity, early training, and learning opportunities, certain individuals will develop some far more than others. The "object-related" intelligences he defined are logical-mathematical (the ability to reason logically, solve numerical problems), spatial (the ability to navigate the environment, form and manipulate mental images), and bodily-kinesthetic intelligence (the ability to carry out motor movement, manipulate objects with finesse). The "object-free" forms of intelligence are verbal (linguistic ability) and musical (the ability to perceive and create pitch and rhythm patterns). Finally, there are two types of personal intelligence: interpersonal (understanding the behavior, thoughts, and feelings of others) and intrapersonal (the ability to understand oneself and develop a sense of one's own identity, “access to one's own feeling life" (Gardner, 1983, p. 241). In his latest book, Gardner (1999) adds a further three possible types of intelligence (naturalistic, spiritual, and existential). The multiple intelligence theory has little or no published empirical evidence, although it has generated a great deal of interest 
among educators (Furnham, 2001). The idea of the specific multiple intelligences proposed (definitely the seven, possibly the ten) seems to "chime" with lay people's understanding of the concept of intelligence. That is, academic tests and theories seem too limited.

More recent studies have examined self-estimates of primary mental abilities as defined by IQ test constructors (Rammstedt \& Rammsayer, 2000, 2001, 2002). These results suggest that the sex difference in estimated IQ is limited to areas measuring mathematical and spatial intelligence.

In order to test the robustness of these findings across cultures, various studies, mainly of the estimate of multiple intelligence, have been done (Zhang \& Gong, 2001). Data has been collected from nearly all the continents; Africa (Uganda), America (United States), Asia (Hong Kong, Japan, Singapore), Europe (Belgium, Germany, Slovakia, United Kingdom) and the Middle East (Iran). These studies have found both sex and culture differences but few interactions between the two. For example Furnham, Hosoe, and Tang (2002) found that, in comparable groups of American, British and Japanese students, the Americans gave themselves highest rating, followed by British, and then by the Japanese.

Table 1 shows the number of countries in which studies have been performed. Most studies have used student participants but some have used school-children and others, non-student adults (Furnham, 2000). With few exceptions, the results have shown: first, that men give higher overall (g) estimates than women; second, that differences are usually restricted to mathematical and spatial intelligence; third, that sex differences in estimates are stronger for self than for others.
However, there have been some interesting and important cultural differences. Some studies, notably from Africa (Furnham \& Akande, 2004) and Eastern Europe (Furnham, Rakow, et al., 1999), have shown that there are fewer than chance sex differences in the self-estimates of intelligence. Second, there appear to be very large differences in the mean scores attributed to self. Thus, studies from Africa show littleeducated mothers giving themselves relatively high scores (over 1.5 standard deviations above the norm), whereas in Asia, well-educated parents and students gave themselves lower scores (less than 0.5 standard deviations above the norm).

As yet, no study has been reported from South America. This study reports on data from Argentina with an Argentinean-British comparison. Argentina, according to Hofstede (1981), has a much higher masculinity score than the United Kingdom (20 vs. 9). It is possible that this leads to exaggerated hubris and larger male versus female differences. However, we hypothesize that:

1. Males will give higher estimates than females on general, as well as on mathematical and spatial intelligence.

2. Participants will rate themselves as significantly more intelligent than their mothers and grandparents but not their fathers.

3. Verbal, mathematical and spatial intelligence will be the only multiple intelligence predictors of overall $g$ intelligence.

4. Test experience, gender, age and education will all be statistically significant predictors of intelligence.

5. Compared to a similar sample from Britain, Argentinean males would give higher self-estimates but lower estimates of parents' IQ.

Table 1

Details of Self-Reported Studies from Sixteen Countries

\begin{tabular}{ll}
\hline Country & Reference \\
\hline America & Furnham, Fong, \& Martin (1999); Hogan, (1978). \\
Belgium & Furnham, Rakow, Sarmany-Schiller, \& De Fruyt (1999) \\
China & Zhang \& Gong (2001) \\
Egypt & Furnham \& Mottabu (2004) \\
Germany & Rammstedt \& Rammsayer (2000, 2001, 2002) \\
United Kingdom & Beloff (1992); Furnham (2000, 2001) \\
Hong Kong & Furnham, Rakow, \& Mak (2002) \\
Iran & Furnham, Shahidi, \& Baluch (2002) \\
Japan & Furnham, Hosoe, \& Tang (2002) \\
Namibia & Furnham \& Akande (2004) \\
New Zealand & Byrd \& Stacey (1993); Furnham \& Ward (2001) \\
Poland & Furnham, Wytykowska, \& Petrides (2004) \\
Singapore & Furnham \& Fong (1999) \\
Slovenia & Furnham, Rakow, Sarmany-Schiller, \& De Fruyt (1999) \\
South Africa & Furnham \& Mkize (2003) \\
Uganda & Furnham \& Baguma (1999) \\
Zimbabwe & Furnham \& Akande (2004) \\
Zambia & Furnham \& Akande (2004) \\
\hline
\end{tabular}


Method

\section{Participants}

A total of 217 participants took part: there were 134 women and 83 men. They ranged in age from 18 to 42 , the modal age being 20 years. Most were single (51.4\%), nearly $31.2 \%$ were married, whereas $14.2 \%$ were living together. Sixty-two percent were studying at the undergraduate level, whereas $13.4 \%$ had a postgraduate degree.

\section{Questionnaire}

Participants completed a one-page questionnaire set out on all previous studies in this area (Furnham, 2000). A normal distribution was shown $(M=100, S D=3)$. Under each standard deviation, a typical IQ score was given plus a descriptor (i.e., " $+1,115$ high average"). Participants were then shown a grid with 8 rows and 6 columns. The first row was labeled "Overall Intelligence" and the remaining 7 were taken from Gardner (1983). There was a short description of what each intelligence stood for (see Table 1). The rows were labeled: you, your partner, your father, mother, grandfather, grandmother. Thus, each participant was requested to make forty-eight IQ estimates. The grid is shown in Table 2. Participants nearly all did estimates by column: that is, they first rated their own overall intelligence with a specific number, then their verbal intelligence, their logical intelligence, etc. They then proceeded to make eleven estimates for their fathers. Apart from standard demographic data, they were also asked if they: (a) believed that people can learn to become more intelligent (27\% responded "yes"), (b) had ever taken an intelligence test (50.9\% responded "yes"), and (c) if they thought intelligence tests measured intelligence fairly well (55\% responded "yes").

\section{Results}

\section{Sex Differences in Estimates}

First a MANCOVA (co-varying out age and education) with sex as the classification factor was computed on each of the six sets of estimates for self-estimates plus partner and four relatives. This was followed by ANCOVAs, first for the overall $g$ score and then for each of the seven multiple intelligences.

Two of the four MANCOVAs were significant: for self, $F(20,209)=6.39, p<.01$, and partner, $F(20,209)=6.82$ $p<.01$. Compared to women, men gave significantly higher estimates for their overall score, $F(1,208)=14.91, p<.001$, mathematical, $F(1,208)=29.84, p<.001$, spatial, $F(1,208)$ $=15.11, p<.001$, and musical intelligence, $F(1,208)=$ $9.18, p<.001$. Effect sizes were, however, relatively small: partial $\eta^{2}=.06, .12, .06$, and .02 , respectively, for overall, mathematical, spatial, and musical intelligence. This confirms the first hypothesis. Similarly men, compared to women, gave lower scores to their "opposite sex" partner on mathematical, $F(1,208)=14.66, p<.001$, and spatial intelligence, $F(1,208)=16.21, p<.001$, but higher scores on intrapersonal intelligence, $F(1,208)=16.21, p<.001$. None of the other four MANCOVAs or the resultant ANCOVAs was statistically significant.

Table 2

Mean (and Standard Deviations) Estimate Scores for Women (W) and Men (M) on each of the Seven Multiple Intelligences for Self and Relatives

\begin{tabular}{lcccccccccccc}
\hline & \multicolumn{2}{c}{ Self } & \multicolumn{2}{c}{ Partner } & \multicolumn{2}{c}{ Father } & \multicolumn{2}{c}{ Mother } & \multicolumn{2}{c}{ Grandfather } & \multicolumn{2}{c}{ Grandmother } \\
\cline { 2 - 15 } & $\mathrm{W}$ & $\mathrm{M}$ & $\mathrm{W}$ & $\mathrm{M}$ & $\mathrm{W}$ & $\mathrm{M}$ & $\mathrm{W}$ & $\mathrm{M}$ & $\mathrm{W}$ & $\mathrm{M}$ & $\mathrm{W}$ & $\mathrm{M}$ \\
\hline \multirow{2}{*}{ OVERALL } & 105.1 & 110.4 & 110.7 & 110.8 & 108.6 & $108.1)$ & 103.4 & 101.1 & 99.0 & 97.1 & 95.9 & 93.9 \\
& $(10.6)$ & $(10.6)$ & $(12.6)$ & $(11.8)$ & $(12.9)$ & $(2.9)$ & $(2.6)$ & $(10.4)$ & $(13.1)$ & $(12.8)$ & $(13.2)$ & $(12.6)$ \\
Verbal & 103.4 & 107.2 & 103.9 & 107.8 & 104.1 & 101.5 & 101.5 & 100.4 & 95.6 & 91.5 & 93.1 & 91.9 \\
& $(13.8)$ & $(15.1)$ & $(14.3)$ & $(14.7)$ & $(15.9)$ & $(18.5)$ & $(14.1)$ & $(13.7)$ & $(14.6)$ & $(14.3)$ & $(13.6)$ & $(17.0)$ \\
Mathematical & 96.9 & 108.3 & 111.6 & 102.9 & 108.2 & 105.8 & 96.0 & 94.6 & 95.9 & 92.6 & 90.8 & 86.3 \\
& $(14.1)$ & $(15.8)$ & $15.3)$ & $(19.2)$ & $(16.3)$ & $(19.3)$ & $(14.5)$ & $(15.0)$ & $(16.8)$ & $(17.8)$ & $(14.1)$ & $(16.0)$ \\
Spatial & 98.8 & 107.9 & 107.7 & 99.3 & 104.3 & 104.0 & 96.7 & 95.5 & 95.6 & 96.4 & 90.7 & 89.7 \\
& $(16.3)$ & $(18.0)$ & $(13.1)$ & $(13.2)$ & $(13.4)$ & $(14.8)$ & $(14.0)$ & $(11.5)$ & $(13.8)$ & $(14.3)$ & $(11.5)$ & $(13.3)$ \\
Musical & 94.9 & 100.3 & 98.5 & 98.9 & 92.1 & 94.6 & 91.5 & 93.1 & 88.8 & 84.7 & 89.6 & 87.1 \\
& $(21.0)$ & $(18.0)$ & $(17.1)$ & $(15.9)$ & $(15.5)$ & $(17.2)$ & $(14.7)$ & $(14.5)$ & $(14.2)$ & $(14.1)$ & $(12.5)$ & $(18.3)$ \\
Body-Kinesthetic & 101.2 & 102.8 & 99.9 & 102.3 & 92.6 & 95.2 & 93.9 & 96.3 & 90.2 & 91.4 & 89.0 & 86.8 \\
& $(15.1)$ & $(14.5)$ & $(15.1)$ & $(14.5)$ & $(12.9)$ & $(17.9)$ & $(12.7)$ & $(13.0)$ & $(13.4)$ & $(14.2)$ & $(12.8)$ & $(15.9)$ \\
Interpersonal & 108.2 & 106.7 & 104.2 & 106.6 & 99.9 & 100.4 & 102.6 & 103.8 & 96.8 & 95.0 & 93.8 & 93.3 \\
& $(13.8)$ & $(15.2)$ & $(14.7)$ & $(15.9)$ & $(16.5)$ & $(17.9)$ & $(15.8)$ & $(15.3)$ & $(18.6)$ & $(16.3)$ & $(16.12)$ & $(18.8)$ \\
Intrapersonal & 104.8 & 108.8 & 100.7 & 107.5 & 96.5 & 95.9 & 99.0 & 99.2 & 94.4 & 93.2 & 94.1 & 92.1 \\
& $(14.7)$ & $(14.7)$ & $(14.2)$ & $(16.1)$ & $(13.9)$ & $(15.9)$ & $(15.4)$ & $(16.3)$ & $(16.8)$ & $(14.5)$ & $(16.3)$ & $(16.9)$ \\
\hline
\end{tabular}




\section{Generational Differences}

A series of paired $t$-tests (with Bonferroni corrections) were performed to examine generational differences. Results are shown in Table 3.

Results show an interesting pattern. Participants gave self and fathers' overall IQ estimates as not significantly different. However, they did believe they were significantly brighter than their mothers (4.59 IQ points), their grandfathers (9.01 IQ points), and grandmothers (12.16 IQ points). This confirms the second hypothesis. They believed their fathers were more intelligent than their mothers (5.85 IQ points), and their grandfathers were brighter than their grandmothers (3.56 IQ points). There was also evidence of a clear generational trend, with each generation being perceived as less intelligent than the previous one (father brighter than grandfather; mother brighter than grandmother, and so on).

\section{Multiple Intelligence Predictors of Intelligence}

In order to find which of the seven multiple intelligences were the best predictors of the overall general score, six multiple regressions were computed with the overall score for self, partner, father, mother, grandfather, and grandmother being the criterion variable, and each of the seven multiple intelligences, respectively, the predictor variable.

The results of the six regressions are fairly consistent. Verbal intelligence was a significant predictor in all six regressions, whereas mathematical and intrapersonal intelligence were significant on five of the six. This provides partial confirmation of the third hypothesis. Musical and interpersonal intelligence were significant in only one regression, whereas body-kinesthetic intelligence was not significant in any of the regressions. The amount of variance accounted for was between 43 and $71 \%$.

Table 3

Paired t-Tests Comparing Overall Estimates of Self and Others

\begin{tabular}{lrrr}
\hline Comparison & \multicolumn{2}{c}{ Mean Scores } & $t$ \\
\hline Self versus Partner & 107.09 & vs. 110.90 & $-4.88 * * *$ \\
Self versus Father & 107.09 & vs. 108.35 & -1.57 \\
Self versus Mother & 107.09 & vs. 102.50 & $5.52 * * *$ \\
Self versus Grandfather & 107.09 & vs. 98.08 & $7.73 * * *$ \\
Self versus Grandmother & 107.09 vs. 94.93 & $11.39 * * *$ \\
Father versus Mother & 108.35 vs. 102.50 & $7.29 * * *$ \\
Father versus Grandfather & 108.35 vs. 98.08 & $7.87 * * *$ \\
Father versus Grandmother & 108.35 vs. 94.93 & $12.53 * * *$ \\
Mother versus Grandfather & 102.50 vs. 98.08 & $3.82 * * *$ \\
Mother versus Grandmother & 102.50 vs. 94.93 & $7.82 * * *$ \\
Grandfather versus Grandmother & 98.16 vs. 94.60 & $3.01 * *$ \\
\hline
\end{tabular}

$* p<.05 . * * p<.01 . * * * p<.001$.

Table 4

Results of the Regression of the Seven Multiple Intelligences onto the Overall Score for Each Set of Estimates

\begin{tabular}{|c|c|c|c|c|c|c|c|c|c|c|c|c|}
\hline & \multicolumn{2}{|c|}{ Self } & \multicolumn{2}{|c|}{ Partner } & \multicolumn{2}{|c|}{ Father } & \multicolumn{2}{|c|}{ Mother } & \multicolumn{2}{|c|}{ Grandfather } & \multicolumn{2}{|c|}{ Grandmother } \\
\hline & $\beta$ & $t$ & $\beta$ & $t$ & $\beta$ & $t$ & $\beta$ & $t$ & $\beta$ & $t$ & $\beta$ & $t$ \\
\hline Verbal & .28 & $5.18 * * *$ & .36 & $5.81 * * *$ & .35 & $5.20 * * *$ & .44 & $7.74 * * *$ & .42 & $7.74 * * *$ & .34 & $4.06 * * *$ \\
\hline Mathematical & .34 & $6.60 * * *$ & .44 & $7.44 * * *$ & .18 & $2.95 * *$ & .21 & $3.87 * * *$ & .14 & 1.58 & .28 & $3.82 * * *$ \\
\hline Spatial & .23 & $4.62 * * *$ & .05 & 0.94 & .13 & $2.24 *$ & .11 & $2.07 *$ & .11 & 1.37 & .08 & 1.16 \\
\hline Musical & .15 & $3.11 * * *$ & .02 & 0.46 & .03 & 0.55 & .04 & 0.66 & .09 & 1.32 & .00 & 0.45 \\
\hline Body-Kinesthetic & .06 & 1.17 & .06 & 1.03 & .04 & 0.69 & .08 & 1.48 & .06 & 0.89 & .09 & 1.41 \\
\hline Interpersonal & .11 & $2.29 *$ & .00 & 0.07 & .02 & 0.22 & .01 & 0.29 & .02 & 0.32 & .08 & 1.09 \\
\hline Intrapersonal & .07 & 1.40 & .13 & $2.16 *$ & .11 & $2.82 * *$ & .16 & $2.66 * *$ & .19 & $2.55 * * *$ & .27 & $3.62 * * *$ \\
\hline$F$ & \multicolumn{2}{|c|}{$40.77 * * *$} & \multicolumn{2}{|c|}{$28.29 * *$} & \multicolumn{2}{|c|}{$23.92 * * *$} & \multicolumn{2}{|c|}{$34.02 * * *$} & \multicolumn{2}{|c|}{$25.11 * * *$} & \multicolumn{2}{|c|}{$49.74 * * *$} \\
\hline Adjusted $R^{2}$ & \multicolumn{2}{|c|}{.56} & \multicolumn{2}{|r|}{.51} & \multicolumn{2}{|c|}{.43} & \multicolumn{2}{|c|}{.52} & \multicolumn{2}{|r|}{.57} & \multicolumn{2}{|c|}{0.71} \\
\hline
\end{tabular}

$* p<.05 . * * p<.01 . * * * p<.001$. 


\section{Predictors of Overall Self-Score}

Two regressions were run to determine to what extent beliefs about intelligence and demography predicted the overall general intelligence estimates. First, answers to the three questions about IQ were regressed onto the total overall estimate. This proved significant, $F(3,207)=3.12, p<.05$, accounting for $3 \%$ of the variance. Those who had previously done an intelligence test gave higher self- estimates, $\beta=.21, t=2.46, p<.01$. Second, sex, age, and educational level plus answers to the three questions were regressed onto the overall estimate of intelligence. This, too, was significant, $F(6,201)=5.93, p<.01$, adjusted $R^{2}=$ .12. It showed that men gave higher self-estimates than women, $\beta=.21, t=3.21, p<.01$, as did older rather than younger people, $\beta=.15, t=2.21, p<.01$, and more- rather than less-educated participants. This provides evidence for the fourth hypothesis.

Table 5

Cross-Cultural Data Showing Argentinean and British Men's and Women's Estimates with 2-Way ANCOVA Results for Self, Partner, Mother and Father

\begin{tabular}{|c|c|c|c|c|c|c|c|}
\hline & \multicolumn{2}{|c|}{ Argentinean } & \multicolumn{2}{|c|}{ British } & \multicolumn{3}{|c|}{$F$ level } \\
\hline & Women & Men & Women & Men & Sex & Culture & Sex x Culture \\
\hline \multicolumn{8}{|l|}{ Self-estimates } \\
\hline Verbal & 103.4 & 107.4 & 109.3 & 113.0 & $5.12 *$ & $12.45^{* * *}$ & 0.02 \\
\hline Mathematical & 96.7 & 108.3 & 105.4 & 116.4 & $43.17 * * *$ & $22.81 * * *$ & 0.00 \\
\hline Spatial & 98.7 & 107.8 & 104.3 & 113.6 & $24.06 * * *$ & $9.20 * *$ & 0.00 \\
\hline Musical & 94.9 & 100.2 & 100.0 & 111.1 & $14.83 * * *$ & 17.42 & 1.47 \\
\hline Body-Kinesthetic & 101.2 & 102.7 & 104.3 & 110.0 & 4.13 & 8.42 & 1.33 \\
\hline Interpersonal & 108.2 & 106.6 & 113.3 & 120.3 & 2.73 & $31.31 * *$ & $6.82 * *$ \\
\hline Intrapersonal & 104.8 & 108.7 & 111.0 & 116.0 & $7.41 * *$ & $17.06 * * *$ & 0.05 \\
\hline \multicolumn{8}{|l|}{ Partner } \\
\hline Verbal & 104.3 & 107.8 & 108.5 & 112.4 & 0.16 & 0.12 & 0.68 \\
\hline Mathematical & 111.9 & 102.9 & 112.5 & 116.5 & $9.34 * *$ & 0.40 & $6.80 * *$ \\
\hline Spatial & 107.2 & 99.3 & 113.1 & 114.5 & $7.52 * *$ & $18.78 * * *$ & $5.13 *$ \\
\hline Musical & 98.4 & 98.9 & 97.0 & 100.1 & 0.60 & $5.03 *$ & 0.00 \\
\hline Body-Kinesthetic & 99.9 & 102.3 & 99.9 & 111.1 & 2.19 & 0.08 & $4.45^{*}$ \\
\hline Interpersonal & 104.2 & 106.6 & 101.1 & 108.6 & 1.53 & $6.51 * *$ & 0.71 \\
\hline Intrapersonal & 99.8 & 107.5 & 105.5 & 111.9 & $5.08 *$ & 0.11 & 1.09 \\
\hline \multicolumn{8}{|l|}{ Father } \\
\hline Verbal & 104.2 & 101.5 & 107.1 & 108.4 & $4.58 *$ & 0.23 & 0.95 \\
\hline Mathematical & 108.3 & 105.8 & 102.8 & 103.6 & $7.48 * *$ & $17.15^{* *}$ & 0.64 \\
\hline Spatial & 104.5 & 104.0 & 102.9 & 105.8 & 1.14 & 2.21 & 0.83 \\
\hline Musical & 92.5 & 93.2 & 98.9 & 108.7 & 0.00 & $9.34 * *$ & $4.81 *$ \\
\hline Body-Kinesthetic & 92.6 & 95.2 & 100.2 & 103.1 & 1.56 & 2.64 & 0.57 \\
\hline Interpersonal & 100.1 & 100.4 & 105.2 & 103.7 & 0.87 & $12.66^{* *}$ & 0.26 \\
\hline Intrapersonal & 96.6 & 95.9 & 108.8 & 112.1 & 0.82 & $27.72 * * *$ & 0.13 \\
\hline \multicolumn{8}{|l|}{ Mother } \\
\hline Verbal & 101.7 & 100.4 & 102.2 & 108.5 & 0.67 & 0.00 & 1.20 \\
\hline Mathematical & 96.3 & 96.4 & 102.2 & 112.1 & 0.79 & $8.64 * *$ & 1.98 \\
\hline Spatial & 96.5 & 95.8 & 105.6 & 116.4 & 0.22 & $31.64 * * *$ & 5.03 \\
\hline Musical & 91.4 & 93.0 & 94.8 & 106.7 & 0.00 & 1.15 & 1.37 \\
\hline Body-Kinesthetic & 94.0 & 96.3 & 95.2 & 106.4 & 1.60 & 0.60 & 1.83 \\
\hline Interpersonal & 102.9 & 103.8 & 96.2 & 104.2 & 0.21 & $16.30 * * *$ & 0.32 \\
\hline Intrapersonal & 99.4 & 99.2 & 102.6 & 105.2 & 0.87 & 0.21 & 0.00 \\
\hline
\end{tabular}

$* p<.05 . * * p<.01 . * * * p<.001$. 


\section{Argentinean versus British Comparison}

Data was available from the United Kingdom that could serve as a comparison given that the samples were of comparable age and sex ratio. The data from Furnham, Shahidi, and Baluch (2002) were used. The British sample had 185 participants of which 129 were female and they were of a comparable age spread and educational level to the Argentinean sample (see Table 5).

Table 6 shows the means for both sexes from the two national groups' (Argentinean and British) estimations of self, partners, and parents, as well as the results of the twoway ANCOVA co-varying out age and education (standard deviations are available from the first author). Six of the seven sex differences for self-estimates were significant and all went in the same direction. In accordance with all the previous literature, men gave higher self-estimates than women, particularly on mathematical and spatial intelligence. Effect sizes varied between .02 and .28 , considering those $F$ levels that reached a $p<.001$ significance level, the effect sizes were all in the region of .2 to .35 . Men also rated their verbal, musical, and intrapersonal intelligence higher than did women. There were far fewer sex differences in the estimates of others: three for partner, two for father, and none for mother.

Results for the main effect for culture/nationality showed many significant differences. All seven main effects were significant for self and all in the same direction: British participants gave higher self-estimates than Argentinean students. The main effect for culture showed five significant for self, three were significant for partner, four for father, and three for mother. With only one exception (ratings of fathers' mathematical intelligence), the British sample gave higher estimates than the Argentineans. There were only six significant interactions and only two at the $p<.01$ level. This indicated that whereas Argentinean men gave lower estimates of their interpersonal intelligence than Argentinean women, it was the opposite for British men, whose mean self-estimates were a full 7 points higher than British women's. The same pattern occurred for the estimate of one's partners' mathematical intelligence.

\section{Discussion}

The results of this study are in line with most of the previous studies in the area. In accordance with studies from nearly all other countries (except those from Africa), men award themselves higher overall IQ scores, as well as mathematical and spatial intelligence (Furnham, 2001) than women. In this study Argentinean men gave themselves higher musical intelligence scores than women, which may be a reflection of differences in music and dance in the Latin versus Anglo-Saxon cultures. However, in previous studies, self-estimates for musical and bodilykinesthetic intelligence were comparatively low (around the mean), whereas self-estimates of inter- and intrapersonal intelligence were around .5 of a standard deviation above the mean.

There were three significant differences for partner ratings. Women rated their male partners as having significantly higher mathematical intelligence than did men of their female partners. Both male and female participants, in fact, gave their partners higher estimates than they gave themselves. A similar result occurred for ratings of spatial intelligence. Also, men rated their female partners as having higher intrapersonal intelligence higher than did women their male partners. In another study of partner ratings, there were similar sex differences in partner estimates (Furnham, Tang, Lester, O'Connor, \& Montgomery, 2002). As before, there were no sex differences in ratings of parents or grandparents (Furnham, Fong, et al., 1999), although other studies did find more evidence of sex differences in the ratings of parents (Furnham, Shahidi, et al., 2002).

In this study, the participants believed they were as bright as their fathers but 6 points brighter than their mothers, 8 points more than their grandfathers, and 11 points more than their grandmothers. There were interesting differences in the estimations of relations: male relations were rated as being brighter than female relations. The results indicated that participants thought each generation was brighter than the last, with the sex differences consistent over the different generations.

Previous studies that regressed the seven multiple intelligences onto the overall intelligence have tended to show that mathematical, spatial, and verbal intelligence are the only significant predictors of overall IQ (Furnham, Tang, et al., 2002). All six regressions were significant (accounting for between 0.43 and $0.71 \%$ of the variance). The results of this study were slightly different in two respects. First, five of the seven self-ratings predicted the overall score including musical and inter personal intelligence. Second, in five of the six regressions, intrapersonal intelligence was a significant predictor. Together with the fact that verbal intelligence was always (and nearly always the most) significant predictor of general intelligence, this suggests that Argentineans have a more literary and emotional conception of general intelligence than do other nations. However, it is important to note that mathematical intelligence was thought to be the single most important predictor for self and partner over each of the other multiple intelligences. Further, in accordance with the previous literature, bodily-kinesthetic intelligence was thought to be unrelated to overall general intelligence.

Data comparing the responses of the Argentinean and British samples were interesting because of the many significant main effects for culture. There was a very clear 
pattern. Almost without exception, the British gave higher estimates than did the Argentineans. Previous studies have illustrated certain national differences in the overall level people tend to estimate intelligence. This Furnham, Hosoe, et al. (2001) showed that with comparable groups of American, British, and Japanese participants, the Japanese gave consistently lower estimates for self and others than those from the other two groups. Similarly, in comparing British and Iranian participants, Furnham, Shahidi, et al. (2001) showed that the latter gave consistently lower estimates than the former. Various factors appear to account for these differences including the levels of education in the countries as well as local norms of hubris and humility about self-presentation.

Studies on self-estimated intelligence have now been carried out on every continent. This is the first from South America. The results show that there are clearly universal patterns in sex differences for self-ratings, beliefs about generational differences, as well as ideas about which of the different multiple intelligences best predicts overall intelligence.

\section{References}

Beloff, H. (1992). Mother, father and me: Our IQ. The Psychologist, 5, 309-311.

Bennett, M. (1997). Self-estimates of ability in men and women. Journal of Social Psychology, 137, 540-541.

Betsworth, D. (1999). Accuracy of self-estimated abilities and the relationship between self-estimated abilities and realism for women. Journal of Career Assessment, 1, 35-43.

Borkenau, P., \& Liebler, A. (1993). Convergence of stranger ratings of personality and intelligence with self-ratings, partner-ratings and measured intelligence. Journal of Personality and Social Psychology, 65, 546-533.

Byrd, M., \& Stacey, B. (1993). Bias in IQ perception. The Psychologist, 6, 16.

Flynn, J. (1987). Massive IQ games in 14 nations: What IQ tests really measure. Psychological Bulletin, 101, 171-191.

Flynn, J. (1999). Searching for justice: The discovery of the gains over time. American Psychologist, 54, 5-20.

Furnham, A. (2000). Parent's estimates of their own and their children's multiple intelligences. British Journal of Developmental Psychology, 18, 583-594.

Furnham, A. (2001). Self-estimates of intelligence: Culture and gender differences in self and other estimates of General (g) and multiple intelligences. Personality and Individual Differences, 31, 1381-1405.

Furnham, A., \& Akande, D. (2004). African parents' estimates of their own and their children's multiple intelligences. Current Psychology, 22, 281-294.

Furnham, A., \& Chamorro-Premuzic, T. (2004). Estimating one's own personality and intelligence scores. British Journal of Psychology, 95, 1-12.
Furnham, A., Clark, K., \& Bailey, K. (1999). Sex differences in estimates of multiple intelligences. European Journal of Personality, 13, 247-259.

Furnham, A., \& Fong, G. (2000). Self-estimated and psychometrically measured intelligence: A cross-cultural and sex difference study. North American Journal of Psychology, 2, 191-199.

Furnham, A., Fong, G., \& Martin, N. (1999). Sex and cross-cultural differences in the estimated multi-faceted intelligence quotient score for self, parents and siblings. Personality and Individual Differences, 26, 1025-1034.

Furnham, A., \& Gasson, L. (1998). Sex differences in parental estimates of their children's intelligence. Sex Roles, 38, 151162.

Furnham, A., Hosoe, T., \& Tang, T. (2001). Male hubris and female humility? A cross- cultural study of ratings of self, parental and sibling multiple intelligence in America, Britain and Japan. Intelligence, 30, 101-115.

Furnham, A., Rakow, T., Sarmany-Schuller, I., \& De Fruyt, F. (1999) European differences in self-perceived multiple intelligence. European Psychologist, 4, 131-138.

Furnham, A., \& Rawles, R. (1995). Sex differences in the estimation of intelligence. Journal of Social Behaviour and Personality, 10, 741-745.

Furnham, A., \& Rawles, R. (1999). Correlations between selfestimated and psychometrically measured IQ. Journal of Social Psychology, 139, 405-410.

Furnham, A., Shahidi, S., \& Baluch, B. (2002). Sex and culture differences in perceptions of estimated multiple intelligences for self and family: A British-Iranian comparison. Journal of Cross-Cultural Psychology, 33, 270-285.

Furnham, A., Tang, T., Lester, D., O'Connor, R., \& Montgomery, R. (2002). Estimates of ten multiple intelligences. European Psychologist, 7, 245-255.

Furnham, A., \& Ward, C. (2001). Sex differences, test experience and the self-estimation of multiple intelligence. New Zealand Journal of Psychology, 30, 52-59.

Gardner, H. (1983). Frames of mind: A theory of multiple intelligences. New York: Basic Books.

Gardner, H. (1999). Intelligence Reframed. New York: Basic Books.

Goodnow, J., \& Collins, W. (1990). Development according to parents: The nature, sources and consequences of parents' ideas. London: Erlbaum.

Hogan, H. (1978). IQ self-estimates of males and females. Journal of Social Psychology, 106, 137-138.

Hofstede, G. (1981). Cultures consequences. New York: Sage.

Paulus, D., Lysy, D., \& Yik, M. (1998). Self-report measures of intelligence: Are they useful as proxy IQ tests? Journal of Personality, 66, 523-555.

Petrides, K., \& Furnham, A. (2000). Gender differences in measured and self-estimated trait emotional intelligence. Sex Roles, 41, 449-461.

Rammstedt, B., \& Rammsayer, T. (2000). Sex differences in selfestimates of different aspects of intelligence. Personality and Individual Differences, 29, 869-880. 
Rammstedt, B., \& Rammsayer, T. (2001). Gender differences is self estimated intelligence in children and early adolescents. German Journal of Educational Psychology, 15, 207-217.

Rammstedt, B., \& Rammsayer, T. (2002). Self-estimated intelligence: Gender differences, relationship to psychometric intelligence and moderating effects of level education. European Psychologist, 7, 275-284.

Reilly, J., \& Mulhern, G. (1995). Gender difference in self-estimated IQ: The need for care in interpreting group data. Personality and Individual Differences, 18, 189-192.
Yuen, A., \& Furnham, A. (in press). Sex differences in selfestimation of multiple intelligences among Hong Kong Chinese Adolescents. High Ability Studies.

Zhang, Y., \& Gong, Y. (2001). Self-estimated intelligence and its related factors. Chinese Journal of Clinical Psychology, 9, 193195.

Received: November 21, 2004 Revision received: December 21, 2004 Accepted: January 26, 2005 